\title{
Value of virtual monochromatic spectral image of dual-layer spectral detector CT with noise reduction algorithm for image quality improvement in obese simulated body phantom
}

Hyo-Jin Kang ${ }^{1,2}$, Jeong Min Lee ${ }^{1,2,3^{*}} \mathbb{D}$, Sang Min Lee ${ }^{4}$, Hyun Kyung Yang ${ }^{5}$, Ri Hyeon Kim ${ }^{1}$ Ju Gang Nam', Aruna Karnawat ${ }^{6}$ and Joon Koo Han 1,2,3

\begin{abstract}
Background: Dual-layer spectral detector CT (SDCT) may provide several theoretical advantages over pre-existing DECT approaches in terms of adjustment-free sampling number and dose modulation, beam hardening correction, and production spectral images by post-processing. In addition, by adopting noise reduction algorithm, high contrast resolution was expected even in low keV level. We surmised that this improvement would be beneficial to obese people. Therefore, our aim of study is to compare image quality of virtual monochromatic spectral images (VMI) and polychromatic images reconstructed from SDCT with different body size and radiation dose using anthropomorphic liver phantom.
\end{abstract}

Methods: One small and one large size of body phantoms, each containing eight (four high- and four lowcontrast) simulated focal liver lesions (FLLs) were scanned by SDCT (at $120 \mathrm{kVp}$ ) using different Dose Right Indexes (DRIs). VMI were reconstructed from spectral base images from $40 \mathrm{keV}$ to $200 \mathrm{keV}$. Hybrid iterative reconstruction $\left(\right.$ iDose $^{4}$ ) was used for polychromatic image reconstruction. Image noise and contrast to noise ratio (CNR) were compared. Five radiologists independently rated lesion conspicuity, diagnostic acceptability and subjective noise level in every image sets, and determined optimal keV level in VMI.

Results: Compare with conventional polychromatic images, VMI showed superior CNR at low keV level regardless of phantom size at every examined DRIs (Ps $<0.05$ ). As body size increased, VMI had more gradual CNR decrease and noise increase than conventional polychromatic images. For low contrast FLLs in large phantom, lesion conspicuities at low radiation dose levels (DRI 16 and 19) were significantly increased in VMI (Ps $<0.05)$. Subjective image noise and diagnostic acceptabilities were significantly improved at VMI in both phantom size.

Conclusions: VMI of dual-layer spectral detector $C T$ with noise reduction algorithm provides improved CNR, noise reduction, and better subjective image quality in imaging of obese simulated liver phantom compared with polychromatic images. This may hold promise for improving detection of liver lesions and improved imaging of obese patients.

Keywords: Dual-energy, Computed tomography, Spectral detector, Phantom, Liver, Obesity

\footnotetext{
* Correspondence: jmsh@snu.ac.kr

'Department of Radiology, Seoul National University Hospital, Seoul 03080,

South Korea

${ }^{2}$ Department of Radiology, Seoul National University College of Medicine,

101 Daehangno, Jongno-gu, Seoul 03080, South Korea

Full list of author information is available at the end of the article
}

(c) The Author(s). 2019 Open Access This article is distributed under the terms of the Creative Commons Attribution 4.0 International License (http://creativecommons.org/licenses/by/4.0/), which permits unrestricted use, distribution, and reproduction in any medium, provided you give appropriate credit to the original author(s) and the source, provide a link to the Creative Commons license, and indicate if changes were made. The Creative Commons Public Domain Dedication waiver (http://creativecommons.org/publicdomain/zero/1.0/) applies to the data made available in this article, unless otherwise stated. 


\section{Background}

Dual-energy CT (DECT) has gained much attention in recent years, and it is frequently used in clinical practice $[1,2]$. Previous reports have been published to demonstrate its clinical utility for evaluation of various abdominal diseases compared with conventional CT, including radiation dose reduction, iodine extraction, increased lesion conspicuity by increasing iodine contrast, reduced image artifacts such as beam-hardening artifacts, and improved tissue and material characterization [1, 3-10]. These advantages of DECT are attributed to the fact that spectral decomposition of DECT data can differentiate intrinsic attenuation related to different atomic numbers and tissue density; whereas conventional polychromatic images from single energy CT cannot [11-18].

Until now, source-based DECT techniques have had disadvantages that include additional radiation exposure and cross beam scattering [1]. Recently, spectral detector CT (SDCT) with a dual-layer based detector has been developed. Using this technique, the superficial detector layer absorbs the lower-energy photons, and the deeper detector layer absorbs the higher-energy photons [6]. The technique may provide several advantages compared to previous DECT approaches [1, 19]. First, it maintains the capability to produce conventional polychromatic images, as well as a variety of spectral post-processed images. Second, since the energy separation is performed by a dual-layer based detector, it is not necessary to adjust the sampling number and dose modulation, ultimately resulting in reduced radiation dose [19-23] and probably not increased radiation dose in obese patients. Third, advanced planning to use the dual energy mode scanning prior to CT examination is not required, which may also provide the advantages of improved workflow. Finally, virtual monochromatic images can be created in the projection (raw data) domain, which have a theoretic quality benefit compared to image-based methods regarding beam-hardening artifact correction [10].

Indeed, several studies have been reported about clinical benefits of SDCT. T. Seller et al. addressed that SDCT deliver more accurate iodine concentration values with higher image contrast than source-based DECT [24]. S. Ehn et al. revealed that SDCT present only small variation (3\%) of iodine concentration with increasing phantom size [25]. In addition, considering the higher contrast resolution of virtual monochromatic images compared to conventional polychromatic images, we surmised that image quality and lesion conspicuity in obese patient could increase without additional radiation exposure using low $\mathrm{keV}$ images from dual-layer spectral detector CT. The purpose of this study was to compare subjective and objective image quality of virtual monochromatic spectral images and polychromatic images reconstructed from dual-layer spectral detector CT
(SDCT) with different body size and radiation dose using anthropomorphic body phantom.

\section{Methods \\ Phantoms}

A customized anthropomorphic abdomen phantom (Kyoto Kagaku Co., Ltd., Kyoto, Japan) containing the liver, pancreas, spleen, kidney, aorta, inferior vena cava, and bones, was used in this study. The phantom size in axial plane was $27 \times 18 \mathrm{~cm}$, which simulated a small body. The craniocaudal length was $30 \mathrm{~cm}$. To compare the image quality of CT images depending on body size, the phantom was wrapped tightly with pork belly subcutaneous fat to emulate a large-sized body $(35 \times 24 \times$ $30 \mathrm{~cm}$ ) (Fig. 1).

According to data from the preliminary phantom study, the attenuation of the organs in this phantom tuned to the portal venous phase. Eight $15-\mathrm{mm}$ spherical focal liver lesions (FLLs) were embedded in the phantom liver. Four of the eight FLLs used were hypovascular simulated lesions with different degrees of lesion-to-liver contrast $(-10,-20,-30$, and $-50 \mathrm{HU})$. The four additional FLLs were hypervascular simulated lesions, with lesion-to-liver contrasts of $+10,+20,+30$, and $+50 \mathrm{HU}$ [26]. Of the eight FLLs, four lesions were considered low contrast lesions $(-20,-10,+10$ and $+20 \mathrm{HU})$ and the other four lesions were considered high contrast lesions ( $-50,-30,+30$ and $+50 \mathrm{HU}$ ) (Fig. 2).

\section{CT image acquisition and reconstruction}

The CT images of the phantom were obtained using dual layer spectral detector CT (IQon, Philips Healthcare, Cleveland, $\mathrm{OH}$ ), which consists of two layered scintillators. The phantom was placed at the isocenter of the gantry with its cross-section perpendicular to the scanner's Z-axis to minimize unnecessary noise. The CT images were obtained at 120 peak kilovoltage (in $\mathrm{kVp}$ ). In every scanning mode, data sets were obtained using the same helical scanning parameters (gantry rotation speed $=0.5 \mathrm{~s} /$ rotation, detector collimation $=0.625 \mathrm{~mm} \times$ 64 slices, helical pitch $=0.797$, field of view $(\mathrm{FOV})=$ $350 \times 350 \mathrm{~mm}$, slice thickness $=3 \mathrm{~mm}$, scan length $=20$ $\mathrm{cm})$. Two different sized phantoms and four different Dose Right Indexes (16, 19, 22, and 25) were used. The Dose Right Index (DRI) is an image-quality reference parameter designed to simplify adjustment of the required image quality specification for the particular diagnostic test [20]. A change in DRI of +1 increases the average tube current by $12 \%$ while decreasing the image noise by $6 \%$ when other factors are unchanged. The mAs values on each $\mathrm{kVp}$, DRI and body size are noted in Table 1.

On this scanner, the obtained data were reconstructed two ways. One is the conventional polychromatic CT 


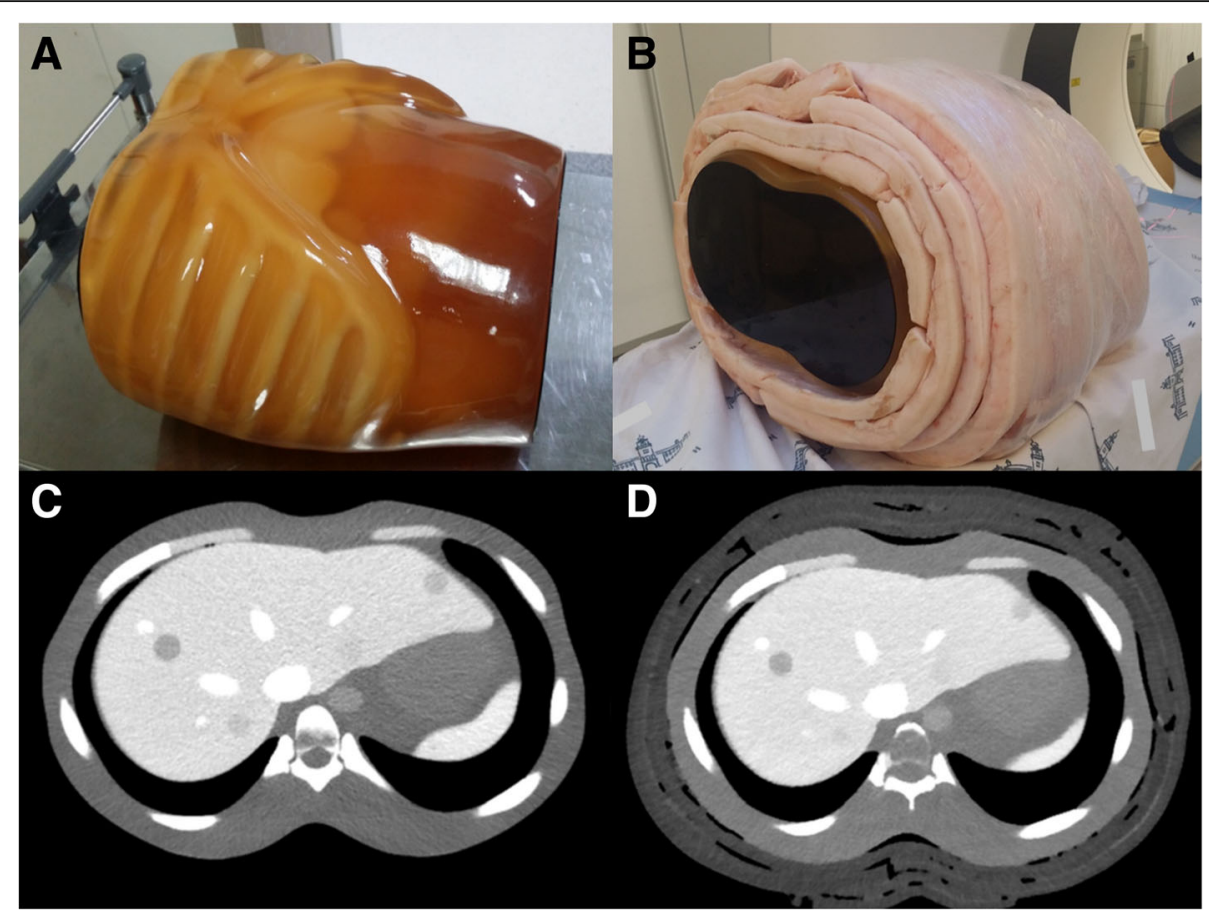

Fig. 1 The appearance of the customized phantom (a) and pork belly-wrapped phantom to mimic a large body size (b). CT images of each phantom size are displayed for (c) small and (d) large body sizes, respectively

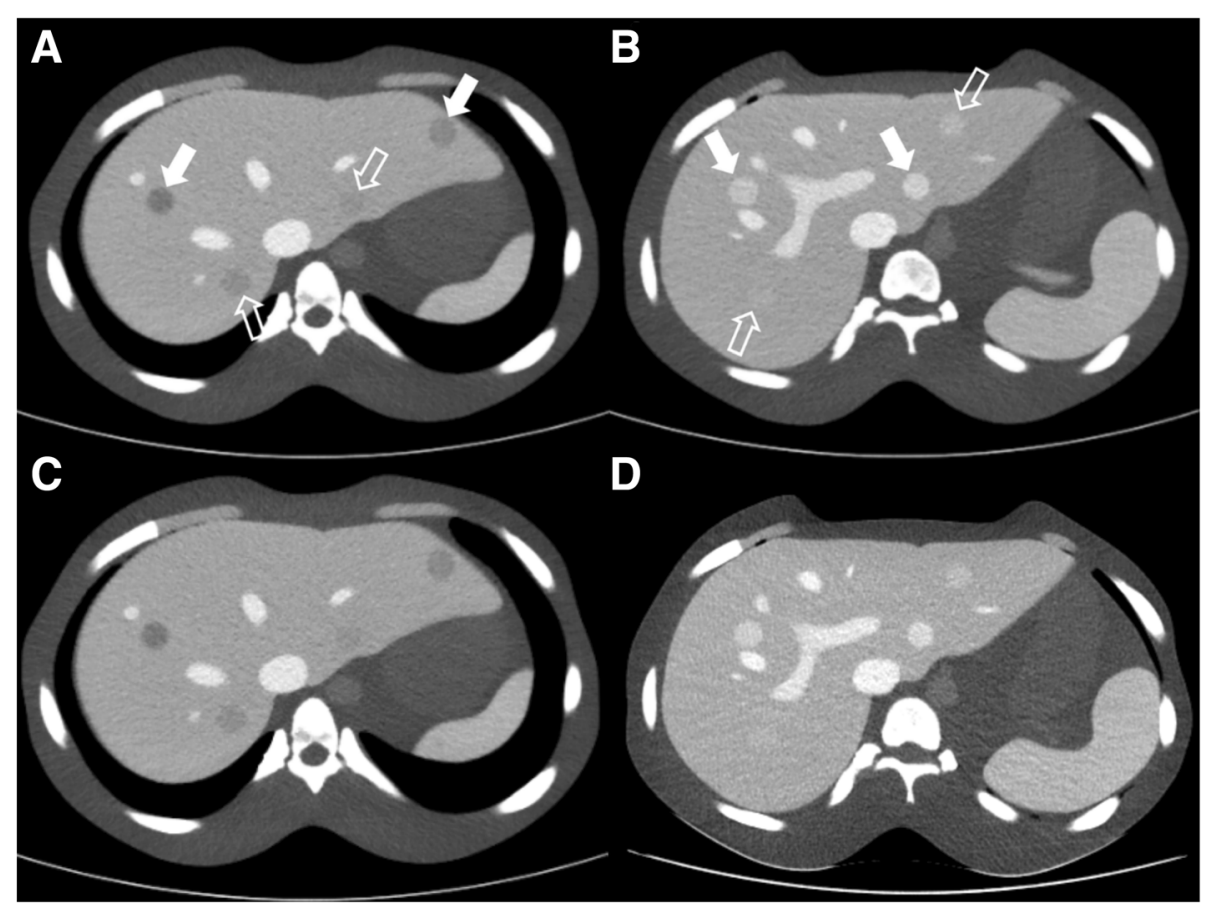

Fig. 2 The eight simulated focal liver lesions (FLLS) in body phantom. a Four hypo-attenuating FLLs are noted. Two were high-contrast hypoattenuating FLLs (white arrows) and other two are low-contrast hypo-attenuating FLLs (empty arrows). b Other four hyper-attenuating FLLs are presented. Likewise, two were high-contrast hyper-attenuating FLLs (white arrows) and other two were low-contrast hyper-attenuating FLLs (empty arrows). The conventional polychromatic images are reconstructed using hybrid iterative reconstruction algorithm (iDose ${ }^{4}$ ) in a level of 4 (a, b). VMI are reconstructed using spectral level 4 and presented in $60 \mathrm{keV}(\mathbf{c}, \mathbf{d})$. All images were applied DRI 19. FLL=focal liver lesion 
Table 1 Radiation doses at different body size and DRI value

\begin{tabular}{llllll}
\hline Size & DRI & mAs & CTDlvol $(\mathrm{mGy})$ & $\mathrm{DLP}(\mathrm{mGy} \times \mathrm{cm})$ & $\mathrm{ED}(\mathrm{mSv})$ \\
\hline $\mathrm{S}(25 \mathrm{~cm})$ & 16 & 60 & 4 & 72 & 1.224 \\
& 19 & 84 & 4.9 & 98 & 1.666 \\
& 22 & 118 & 6.7 & 134 & 2.278 \\
& 25 & 165 & 9.6 & 192 & 3.264 \\
$\mathrm{~L}(35 \mathrm{~cm})$ & 16 & 97 & 5.2 & 104 & 1.768 \\
& 19 & 135 & 7.3 & 146 & 2.482 \\
& 22 & 190 & 10.3 & 206 & 3.502 \\
& 25 & 266 & 14.4 & 288 & 4.896 \\
\hline
\end{tabular}

Note - DRI Dose Right Index, CTDIvol computed tomography dose index volume, DLP dose length product, $E D$ effective dose

image that was reconstructed from combined data of two detector layers with following de-noising process. The other is the virtual monochromatic spectral image that use data from each detector layer, separately. In decomposition step of raw CT data, scattering and photoelectric absorption diagram were used for material separation, and generating spectral results [27]. De-noising process were done in pre- and post-decomposition step.

Scanned polychromatic data were reconstructed using a hybrid iterative reconstruction algorithm (iDose ver. 4, Philips Healthcare, Amsterdam, Netherlands) with a level of 4 , obtained by blending approximately $50 \%$ filtered back projection (FBP) and 50\% iterative reconstruction (IR) for clinical interpretation [28], and the reconstruction level of 4 were recommend by vendor. VMI were reconstructed at level 4 (medium), which was selected among the seven levels (level 1, lowest; level 7, highest) according to vendor's recommendation. The vendor specific noise reduction algorithm such as anticorrelateive filter, structure propagation and constrained noise suppression were automatically applied and spectral reconstruction level implies a degree of noise reduction. Without pre- and post-processing step, images were displayed on a vendor-specific workstation (Philips IntelliSpace Portal, Philips Healthcare, Amsterdam, Netherlands) using four different DRIs, as were the conventional images.

\section{Radiation dose}

DRI is a value derived from commercially available dose modulation program (Philips Healthcare, Amsterdam, Netherlands). Theoretically, scan images with the same DRI will also have the same computed tomography dose index (CTDI) value if $\mathrm{kVp}$ and body size are equal. The DRI, computed tomography dose index volume (CTDIvol), and dose length product (DLP) were recorded, and the effective dose (ED) was calculated by multiplying by the tissue conversion factor of the abdomen $(0.017$ $\mathrm{mSv} \times \mathrm{mGy}^{-1} \times \mathrm{cm}^{-1}$ ) [29]. More detailed information of radiation dose depending on body size and DRI are presented in Table 1.

\section{Quantitative analysis}

Three circular regions of interest (ROIs) were drawn on the anterior abdominal wall and bilateral paraspinal muscle layers at the level of T9 vertebral body of every phantom image to evaluate the image noise (mean, $165.9 \mathrm{~mm}^{2}$; range, $109.2-217.6 \mathrm{~mm}^{2}$ ). Each measurement was repeated three times to ensure consistency, and the average of the standard deviation of each measurement was considered image noise. The attenuation of the liver parenchyma (mean, $174.9 \mathrm{~mm}^{2}$; range, 125.5-226.1 $\mathrm{mm}^{2}$ ) was measured at the left lateral segment, right anterior segment, and right posterior segment at the level of the T9 vertebral body. The attenuation of a most hyper-attenuated FLL (mean, $62.1 \mathrm{~mm}^{2}$; range, 46.0$81.4 \mathrm{~mm}^{2}$ ) and hypo-attenuating FLL (mean, $60.2 \mathrm{~mm}^{2}$; range, $53.0-83.6 \mathrm{~mm}^{2}$ ) were measured three times. The representative image were presented in Fig. 3. A fellowship trained body radiologist (H.J.K, seven years of experience in abdominal radiology) performed all measurements.

The lesion-to-liver CNR was calculated using the following formula:

$$
\mathrm{CNR}=\left(\mathrm{ROI}_{\text {lesion }}-\mathrm{ROI}_{\text {liver }}\right) / \mathrm{SD}_{\text {noise }}
$$

The lesion ROI and liver ROI were the mean attenuations of the liver nodule and parenchyma, respectively. Noise SD was the mean of image noise which was defined as the standard deviation of the attenuation values measure in the phantom's background.

\section{Qualitative analysis}

Five radiologists (S.M.L., H.K.Y., Aruna, L.H.K., and J.K.N. with $10,7,10,6$, and 4 years of experience in abdominal imaging, respectively) analyzed the qualitative phantom image quality regarding image noise, diagnostic acceptability, and lesion conspicuity of each FLL. They were allowed to change window and level as well as keV (up to $110 \mathrm{keV}$ ) level to find best interpretable images and asked to record used $\mathrm{keV}$ level in each image stack. Image noise was evaluated using a 5-point scale based on consensus from previous studies $[26,30,31]$ as follows: 1 = unacceptable; 2 =above average; 3 =average; $4=$ below average; $5=$ minimal or absent. Diagnostic acceptability was graded on a 5-point scale as follows: $1=$ diagnostically unacceptable; 2 = suboptimal for diagnosis; $3=$ average; $4=$ above average; $5=$ excellent. Lesion conspicuity of each of FLL was assessed on a 5-point scale as follows: $1=$ not detectable; $2=$ barely delineated; $3=$ average contrast, margin not round and blurry; $4=$ 


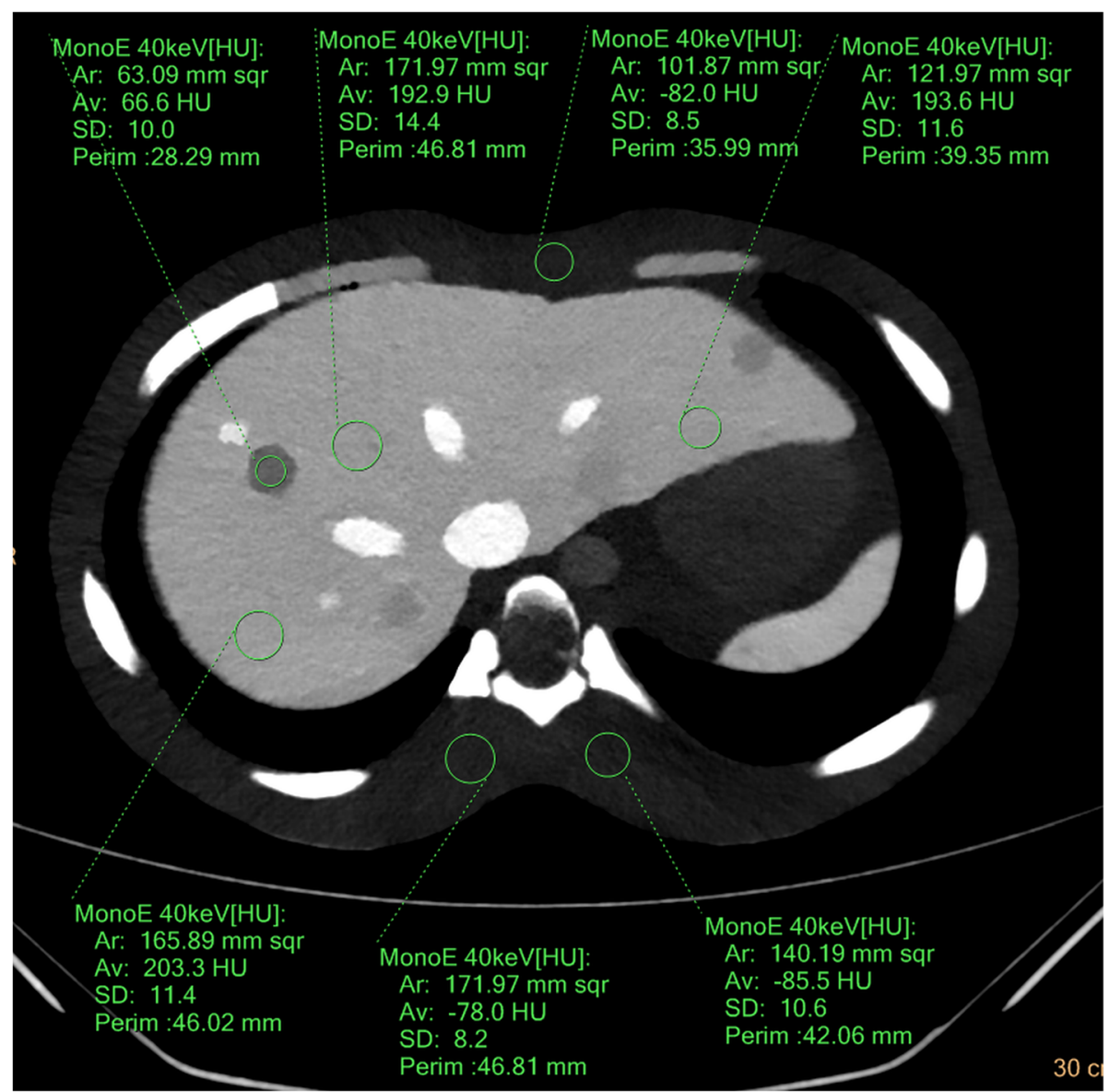

Fig. 3 An axial virtual monochromatic image that show ROls manually drawn on anterior abdominal wall, bilateral paraspinal muscle, liver parenchyma and a hypo-attenuating FLL. Other ROls drawn in other axial image (not shown) to measure the attenuation of hyper-attenuating FLLs

relatively good contrast, margin round but blurry; $5=$ good contrast, margin distinct and round.

\section{Statistical analysis}

Continuous data (noise and CNR) was analyzed using a paired t-test. Diagnostic acceptability and image noise were compared using repeated-measure analysis of variance (ANOVA) and post hoc analysis. To compare the lesion conspicuity among the polychromatic and virtual monochromatic images, the paired T-test was performed. All statistical analysis was performed using commercially available software (SPSS version 22, IBM Corporation, Armonk, NY, USA). Two-tail $p$-values less than 0.05 were considered statistically significant.

\section{Results}

\section{Quantitative analysis}

\section{Attenuation and noise}

Phantom size and DRI values did not make a significant attenuation differences in VMI as well as polychromatic images, while attenuations of the liver parenchyma decreased as $\mathrm{keV}$ increased in VMI. When the DRI value is same, noise of VMI were lower than polychromatic images (all $P<0.05$ ) except low keV range (Fig. 4a). All the mean noise and CNR values are described in Table 2.

\section{CNR of hypo- and hyper-attenuating FLLs}

Compared to polychromatic images, the CNR values of hyper-attenuating FLLs in VMI were higher at low keV range in every DRI values (DRI 16, range $40-79 \mathrm{keV}$; DRI 19, range 40-75 keV; DRI 22, range $40-81 \mathrm{keV}$; DRI 25, 40-92 keV) (Fig. 4b). Similarly, the CNR value of hypo-attenuating FLL of VMI were higher at low keV range than that of polychromatic image in each DRI values (range of $40-80 \mathrm{keV}$ on DRI $16,40-83 \mathrm{keV}$ on DRI $19,40-88 \mathrm{keV}$ on DRI $22,40-90 \mathrm{keV}$ on DRI 25) (Fig. 4c). The CNR values of hypo- and hyper-attenuating FLLs were the highest at VMI $40 \mathrm{keV}$ and gradually decreased as $\mathrm{keV}$ increased. 

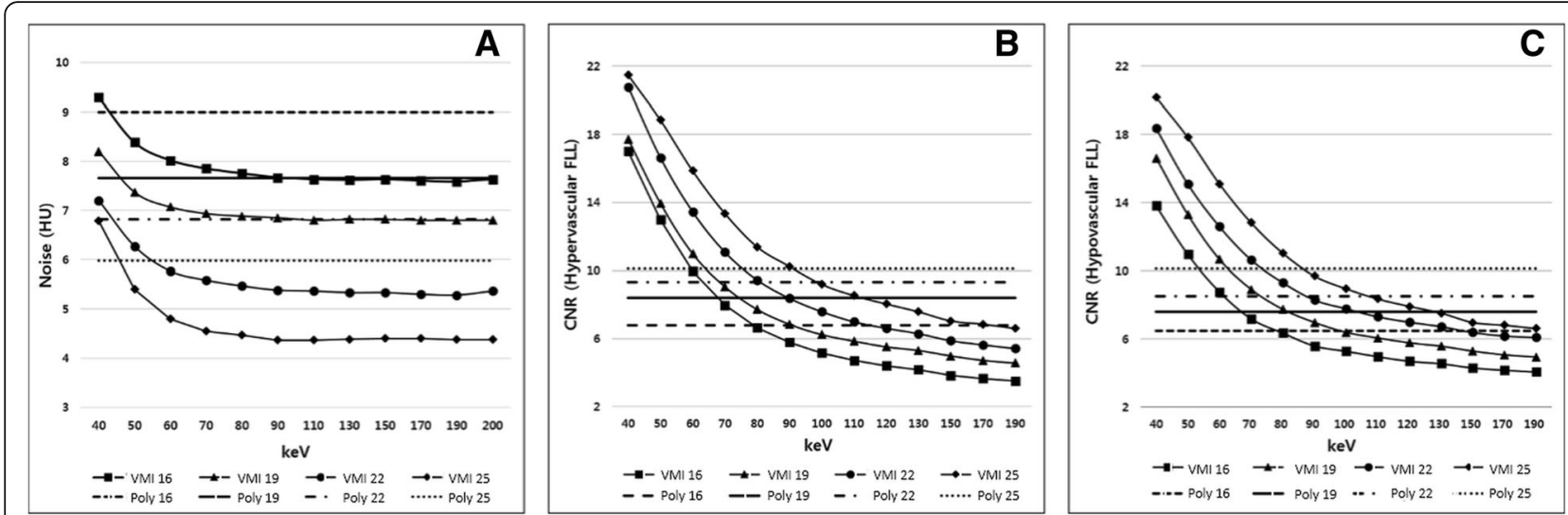

Fig. 4 The graphs show the mean noise in the (a) polychromatic images and VMI in small phantom. When the same radiation dose level (same DRI), noise of VMI were lower than polychromatic images (all $P<0.05$ ) except low keV range. The graph $(\mathbf{b})$ and $(\mathbf{c})$ presented the CNR of $(\mathbf{b})$ hyper- or (c) hypo-attenuating FLL in polychromatic images and VMI of the small phantom. The CNR value gradually decreases as keV increases and has a higher value in low keV ranges than that of the polychromatic images with equal DRI values. DRI dose right index, VMI virtual monochromatic spectral image, Poly polychromatic image

\section{Image noise and CNR in different body sizes}

When the body size increased, polychromatic image showed stiffer noise increment than VMI. Thus, the difference of the noise between VMI and polychromatic images was increased in large phantom (Fig. 5). The maximum differences of noise were 1.4 HU observed in the small phantom and 2.4 HU in the large phantom, with a DRI 16. For a DRI 19, the maximum noise differences were $0.9 \mathrm{HU}$ in the small phantom and $2.1 \mathrm{HU}$ in the large phantom. With a DRI of 22, maximum observed differences were
$1.5 \mathrm{HU}$ in the small phantom and 1.6 HU in the large phantom. As a result, VMI had more gradual CNR decrease and noise increase than conventional polychromatic images (Figs. 6 and 7).

\section{Qualitative analysis \\ Diagnostic acceptability and subjective image noise}

The subjective image quality scores are summarized in Table 3. In small phantom, diagnostic acceptability

Table 2 Noise and CNR values of polychromatic and VMls with different DRIs, body sizes, and energy

\begin{tabular}{|c|c|c|c|c|c|c|c|c|c|c|c|c|c|c|c|c|c|c|c|c|}
\hline \multirow{2}{*}{$\begin{array}{l}\text { Body } \\
\text { size }\end{array}$} & & \multirow[t]{2}{*}{ DRI } & \multirow{2}{*}{$\begin{array}{l}\text { Polychromatic } \\
\text { image }\end{array}$} & \multicolumn{17}{|c|}{ VMI (keV) } \\
\hline & & & & 40 & 45 & 50 & 55 & 60 & 65 & 70 & 75 & 80 & 90 & 100 & 110 & 120 & 140 & 160 & 180 & 200 \\
\hline \multirow[t]{8}{*}{ S } & Noise & 16 & 8.99 & 9.30 & 8.78 & 8.38 & 8.18 & 8.02 & 7.90 & 7.85 & 7.78 & 7.75 & 7.67 & 7.65 & 7.63 & 7.63 & 7.63 & 7.62 & 7.62 & 7.63 \\
\hline & & 19 & 7.66 & 8.20 & 7.67 & 7.37 & 7.20 & 7.07 & 6.95 & 6.93 & 6.90 & 6.88 & 6.85 & 6.87 & 6.80 & 6.83 & 6.83 & 6.77 & 6.78 & 6.80 \\
\hline & & 22 & 6.81 & 7.20 & 6.63 & 6.27 & 5.98 & 5.77 & 5.62 & 5.58 & 5.52 & 5.47 & 5.38 & 5.37 & 5.37 & 5.33 & 5.35 & 5.33 & 5.33 & 5.37 \\
\hline & & 25 & 5.98 & 6.78 & 6.00 & 5.40 & 5.05 & 4.80 & 4.68 & 4.55 & 4.47 & 4.47 & 4.37 & 4.38 & 4.37 & 4.35 & 4.38 & 4.42 & 4.43 & 4.42 \\
\hline & CNR & 16 & 6.78 & 17.00 & 14.82 & 12.96 & 11.27 & 9.95 & 8.86 & 7.96 & 7.23 & 6.63 & 5.78 & 5.15 & 4.71 & 4.40 & 3.96 & 3.70 & 3.53 & 3.41 \\
\hline & & 19 & 8.39 & 17.72 & 15.82 & 13.95 & 12.29 & 10.98 & 9.96 & 9.05 & 8.29 & 7.72 & 6.85 & 6.23 & 5.85 & 5.51 & 5.11 & 4.90 & 4.73 & 4.64 \\
\hline & & 22 & 9.33 & 20.76 & 18.68 & 16.63 & 14.91 & 13.44 & 12.24 & 11.07 & 10.15 & 9.45 & 8.37 & 7.59 & 6.99 & 6.61 & 6.04 & 5.71 & 5.50 & 5.34 \\
\hline & & 25 & 10.90 & 21.48 & 20.15 & 18.85 & 17.27 & 15.86 & 14.37 & 13.35 & 12.32 & 11.38 & 10.24 & 9.19 & 8.53 & 8.04 & 7.33 & 6.83 & 6.56 & 6.40 \\
\hline \multirow[t]{8}{*}{ L } & Noise & 16 & 12.36 & 11.43 & 11.27 & 10.90 & 10.62 & 10.50 & 10.28 & 10.22 & 10.18 & 10.12 & 9.98 & 9.98 & 9.98 & 9.93 & 9.92 & 9.87 & 9.88 & 10.10 \\
\hline & & 19 & 10.59 & 10.18 & 9.70 & 9.35 & 9.12 & 8.93 & 8.85 & 8.73 & 8.68 & 8.67 & 8.60 & 8.57 & 8.52 & 8.53 & 8.47 & 8.48 & 8.42 & 8.45 \\
\hline & & 22 & 9.44 & 8.47 & 7.98 & 7.68 & 7.42 & 7.33 & 7.18 & 7.15 & 7.05 & 7.07 & 6.97 & 6.92 & 6.92 & 6.95 & 6.90 & 6.90 & 6.85 & 6.90 \\
\hline & & 25 & 4.32 & 6.82 & 6.37 & 6.15 & 6.03 & 5.90 & 5.82 & 5.78 & 5.78 & 5.75 & 5.73 & 5.73 & 5.72 & 5.72 & 5.70 & 5.75 & 5.68 & 5.73 \\
\hline & CNR & 16 & 4.41 & 11.56 & 9.82 & 8.63 & 7.67 & 6.83 & 6.23 & 5.71 & 5.25 & 4.92 & 4.44 & 4.07 & 3.80 & 3.62 & 3.38 & 3.23 & 3.12 & 2.99 \\
\hline & & 19 & 5.46 & 14.69 & 12.69 & 11.00 & 9.56 & 8.40 & 7.44 & 6.73 & 6.07 & 5.55 & 4.82 & 4.30 & 3.94 & 3.66 & 3.31 & 3.11 & 2.95 & 2.87 \\
\hline & & 22 & 6.30 & 17.78 & 15.63 & 13.68 & 12.12 & 10.67 & 9.64 & 8.72 & 8.02 & 7.38 & 6.52 & 5.94 & 5.50 & 5.13 & 4.73 & 4.46 & 4.39 & 4.87 \\
\hline & & 25 & 7.82 & 20.87 & 18.56 & 16.21 & 14.20 & 12.69 & 11.38 & 10.35 & 9.45 & 8.73 & 7.69 & 6.97 & 6.51 & 6.13 & 5.63 & 5.25 & 5.09 & 4.98 \\
\hline
\end{tabular}



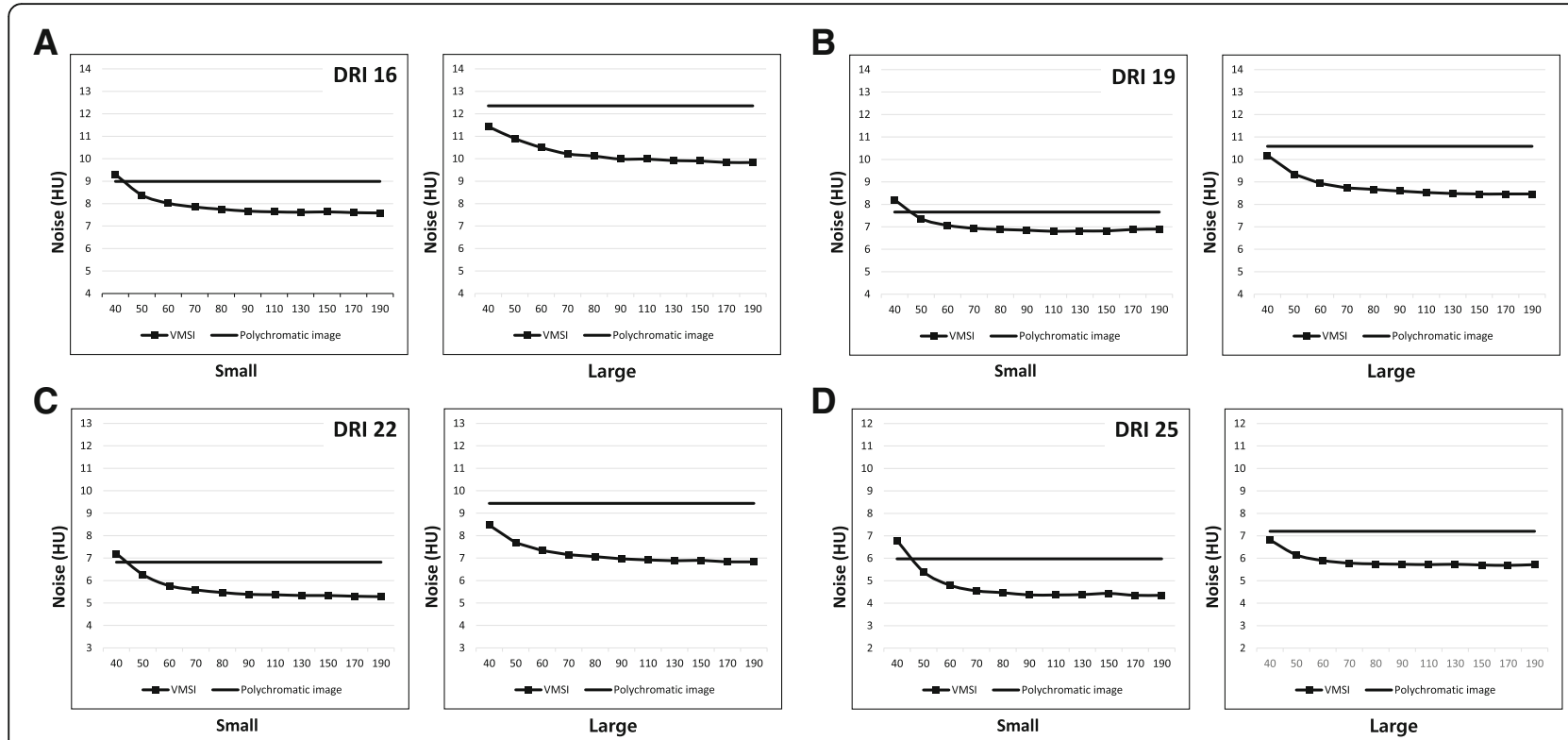

Fig. 5 Image noise of polychromatic image and VMI in small and large phantoms. The maximum noise gap increases as phantom size increases in all examined DRIs ([a] DRI 16, [b] DRI 19, [c] DRI 22, [d] DRI 25). DRI = dose right index, VMI virtual monochromatic spectral image, DRI dose right index

and subjective noise score were significantly improved in VMI compared to polychromatic images $(P=0.02$ and $P<0.01$, respectively). In large phantom, VMI presented superior diagnostic acceptability and subjective noise score than polychromatic images $(P=$ 0.03 and $P=0.04$, respectively).

\section{Lesion conspicuity of FLLs}

The lesion conspicuities of FLLs in large phantom (combination of hypo- or hyper-attenuating and low or high contrast FLLs; all eight FLLs) on VMI were generally higher than that of polychromatic images (Table 4). The differences were more evident in the low-contrast FLLs
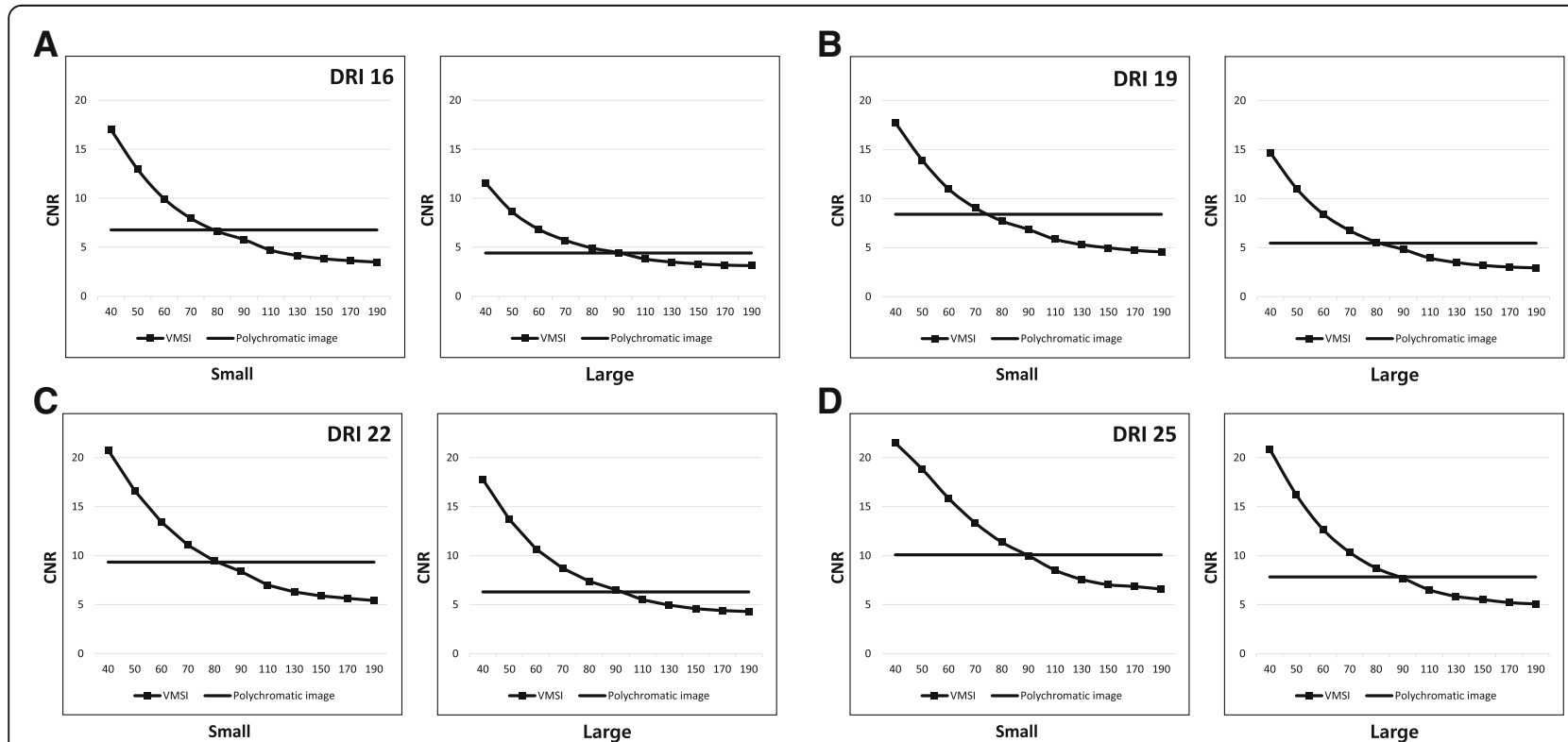

Fig. 6 CNR values of polychromatic image and VMI in small and large phantoms. VMI had more gradual CNR decrease than conventional polychromatic images in all examined DRIs ([a] DRI 16, [b] DRI 19, [c] DRI 22, [d] DRI 25). DR/ dose right index, VMI virtual monochromatic spectral image, $D R /$ dose right index 


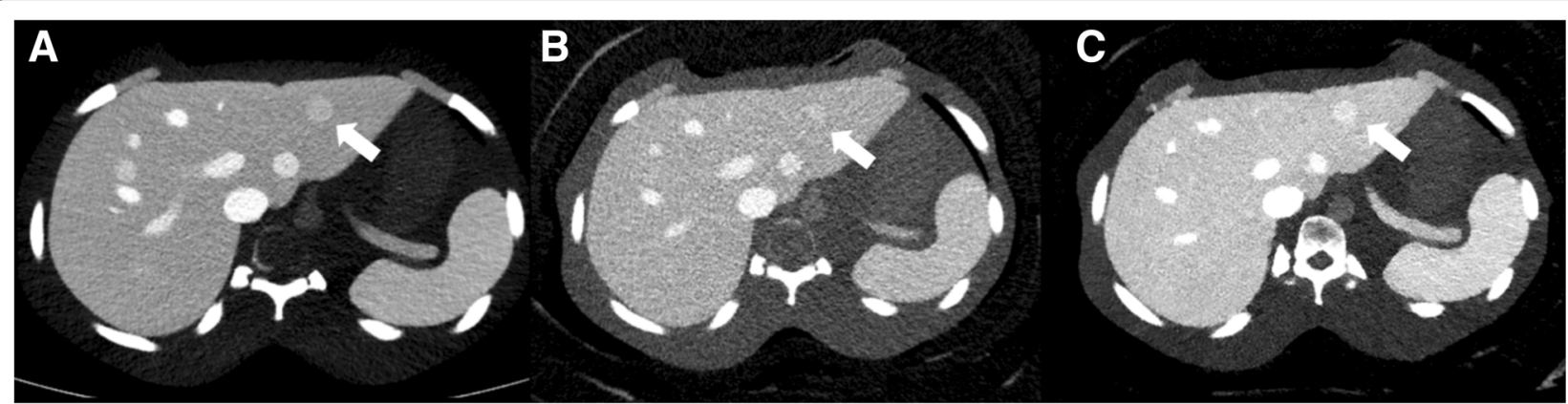

Fig. 7 Low-contrast hyper-attenuating FLL (arrow) in the left lateral segment of the liver with different body phantom sizes. On polychromatic iDose ${ }^{4}$ image, as the phantom size is bigger ([a] small and [b] large), the low-contrast FLL is less visible. When adjusting the image for a low keV level (56 keV) VMI on large size phantom (c), the FLL visibility is markedly improved compared polychromatic iDose ${ }^{4}$ images. FLL focal liver lesion, $D R /$ dose right index, VMI virtual monochromatic spectral image

$(P=0.01$ for DRI 16 and $P=0.02$ for DRI 19). Representative examples are presented in Fig. 7.

\section{Discussion}

Compared to polychromatic images, we found that subjective image noise score and diagnostic acceptability were improved in both small and large phantoms using VMI. In addition, when the phantom size increased, VMI had more gradual CNR decrease and noise increase than conventional polychromatic images. We can attribute this improvement in image quality to effective noise reduction and the superior CNR of dual-layer spectral detector CT, especially in low energy level. Indeed, lesion conspicuity of low contrast FLLs was significantly increased in VMI, compared with polychromatic images.

Our study results were in good agreement with previous studies of DECT using a new dual-source CT scanner [32-36]. Accumulating evidence suggests that optimized virtual monochromatic images $(60 \sim 70 \mathrm{keV})$ with the lowest noise in the reconstructed monochromatic dataset can improve image quality compared with a conventional single energy $\mathrm{CT}$ technique with the same radiation dose $[1,3,10,33,37]$. One thing different from previous studies is optimal $\mathrm{keV}$ range in VMI. Our study resulted demonstrated that VMI had lower image noise, and higher CNR values at low energy ranges (40 to $75 \sim 95 \mathrm{keV}$ for hyper-attenuating FLL; 40 to $80 \sim 90 \mathrm{keV}$ for hypo-attenuating FLL) compared with polychromatic images. However, previous studies using the earlier dual-source DECT technique or fast-switching voltage DECT techniques demonstrated that the gain in CNR seen in low energy level virtual monochromatic images is counterbalanced by a substantial increase in image noise, compared with a single energy scan. Therefore, a range of 60 to 70 $\mathrm{keV}$ (similar to $120 \mathrm{kVp}$ ) was suggested as the optimal energy level $[15,32,33,38]$. The improved CNR values of the VMI taken at low energy ranges could be attributed to the increased attenuation of iodinated contrast agents and to vendor-specific noise reduction algorithms, including mitigation of anti-correlative noise after decomposition. In our study, VMI of duallayer spectral detector CT system allowed the use of lower energy to benefit from the increased iodine CNR, without increasing the image noise at the same radiation dose level used in conventional single-energy CT. The elevated CNR would be beneficial for lesion detection in the abdominal solid organs,

Table 3 Quality assessments of polychromatic and VMIs using variable DRI values in small and large phantoms

\begin{tabular}{|c|c|c|c|c|c|c|c|c|c|}
\hline & & \multicolumn{4}{|c|}{ Diagnostic acceptability } & \multicolumn{4}{|c|}{ Subjective noise } \\
\hline & & DRI 16 & DRI 19 & DRI 22 & DRI 25 & DRI 16 & DRI 19 & DRI 22 & DRI 25 \\
\hline \multirow[t]{3}{*}{$S$} & Polychromatic & $3.0(0.00)$ & $3.2(0.45)$ & $3.2(0.84)$ & $4.0(1.00)$ & $3.3(0.50)$ & $3.8(0.50)$ & $4.0(0.00)$ & $4.5(0.58)$ \\
\hline & $\left.\mathrm{VM}\right|^{*}$ & $3.4(1.10)$ & $4.0(1.00)$ & $4.4(0.89)$ & $4.6(0.55)$ & $3.5(0.58)$ & $4.5(0.58)$ & $5.0(0.00)$ & $5.0(0.00)$ \\
\hline & $p$ value & $0.02+$ & & & & $<0.01 \dagger$ & & & \\
\hline \multirow[t]{3}{*}{$\mathrm{L}$} & Polychromatic & $2.5(0.55)$ & $3.0(0.50)$ & $3.4(0.58)$ & $4.0(0.57)$ & $2.0(0.00)$ & $3.3(0.50)$ & $4.0(0.00)$ & $5.0(0.00)$ \\
\hline & $\mathrm{VMI}^{*}$ & $2.8(0.96)$ & $3.8(0.45)$ & $4.2(0.88)$ & $4.6(0.55)$ & $2.5(0.45)$ & $3.8(0.50)$ & $4.5(0.58)$ & $5.0(0.00)$ \\
\hline & $p$ value & $0.03+$ & & & & $0.04 \dagger$ & & & \\
\hline
\end{tabular}

Note - * VMI were evaluated in range of 40-110 keV. Data presented with † indicate those with statistical significance. Values given are mean (SD). DRI dose right index, $S$ small, $L$ large 
Table 4 Quality assessment of lesion conspicuity of 8 FLLs at polychromatic and VMIs with variable DRI value in large phantom

\begin{tabular}{|c|c|c|c|c|c|c|c|c|}
\hline & \multicolumn{4}{|c|}{ High contrast FLLs $(n=160)$} & \multicolumn{4}{|c|}{ Low contrast FLLs $(n=160)$} \\
\hline & DRI $16(n=40)$ & DRI $19(n=40)$ & DRI $22(n=40)$ & DRI $25(n=40)$ & DRI $16(n=40)$ & DRI $19(n=40)$ & DRI $22(n=40)$ & DRI $25(n=40)$ \\
\hline & \multicolumn{8}{|l|}{ Overall $(n=320)$} \\
\hline Polychromatic & $4.5(0.76)$ & $4.4(0.82)$ & $4.4(0.82)$ & $4.55(0.76)$ & $2.5(0.89)$ & $2.8(0.95)$ & $2.85(1.0)$ & $3.15(1.14)$ \\
\hline VMlt & $4.6(0.68)$ & $4.6(0.76)$ & $4.6(0.68)$ & $4.6(0.68)$ & $3.0(1.00)$ & $3.3(1.16)$ & $3.2(1.3)$ & $3.4(1.23)$ \\
\hline \multirow[t]{2}{*}{$P$-value } & 0.08 & 0.18 & $0.04^{*}$ & 0.56 & $0.01^{*}$ & $0.02^{*}$ & 0.05 & 0.06 \\
\hline & \multicolumn{8}{|c|}{ Hyper-attenuating $(n=160)$} \\
\hline Polychromatic & $4.4(0.84)$ & $4.5(0.85)$ & $4.5(0.85)$ & $4.6(0.84)$ & $2.4(1.1)$ & $2.7(1.06)$ & $2.8(1.23)$ & $3.2(1.14)$ \\
\hline VMl† & $4.6(0.7)$ & $4.6(0.7)$ & $4.6(0.7)$ & $4.6(0.7)$ & $2.9(1.1)$ & $3.2(1.14)$ & $3.2(1.31)$ & $3.4(1.27)$ \\
\hline \multirow[t]{2}{*}{$P$-value } & 0.16 & 0.32 & 0.32 & 1 & 0.59 & 0.06 & $0.04^{*}$ & 0.31 \\
\hline & \multicolumn{8}{|c|}{ Hypo-attenuating $(n=160)$} \\
\hline Polychromatic & $4.5(0.71)$ & $4.3(0.82)$ & $4.3(0.82)$ & $4.5(0.71)$ & $2.6(0.7)$ & $2.7(0.88)$ & $2.9(0.88)$ & $3.2(1.35)$ \\
\hline VMlt & $4.6(0.7)$ & $4.5(0.85)$ & $4.6(0.7)$ & $4.6(0.69)$ & $3.1(0.99)$ & $3.3(1.25)$ & $3.2(1.4)$ & $3.4(1.27)$ \\
\hline$P$-value & 0.32 & 0.32 & 0.08 & 0.32 & 0.06 & 0.16 & 0.28 & 0.16 \\
\hline
\end{tabular}

Note - Data presented with * indicate those with statistical significance. Values given are mean (SD). + VMI were evaluated in range of $40-110$ keV. VMI virtual monochromatic spectral image, $D R I$ dose right index, FLL focal liver lesion

including hepatocellular carcinoma or pancreatic tumors; also, low-energy VMI from dual-layer spectral detector CT may provide additional benefit such as radiation dose reduction compared with polychromatic images.

In terms of radiation dose reduction, VMI technique may be used to reduce radiation dose considering the higher CNR values observed in images collected at low energy ranges. The CNR values of 40, 50 and $60 \mathrm{keV}$ VMIs with a DRI 16 was higher than a polychromatic image with a DRI 19. The results were due to the property of iodine accentuation, and they suggest that images with lower DRI values with VMI reconstruction are not inferior to conventional CT images with higher DRI values taken at a low energy range, which offers sufficient diagnostic image quality. In addition, new technologic for noise reduction such as discriminative feature representation (DFR) would be a promising tool for further noise reduction especially in low $\mathrm{keV}$ range [39-41].

Phantom size notably affected the CNR and noise in both polychromatic and virtual monochromatic images collected from dual-layer spectral detector CT. As body size increased, VMI had more gradual CNR decrease and noise increase than conventional polychromatic images. The CNR and noise value differences in polychromatic and virtual monochromatic images were greater in large phantom compared with small phantoms (max. Noise gaps in small phantom: 1.4 HU, large phantom: 2.4 HU; higher $\mathrm{CNR}<78 \mathrm{keV}$ in small phantom, higher $\mathrm{CNR}<82 \mathrm{keV}$ in large phantom). These results may be due to the fact that a polychromatic beam possess a wide energy spectrum; the energy spectrum is more hardened when passing through a larger phantom as the attenuation of lower energy X-rays is higher than that of high-energy X-rays $[4,7,42]$. Although the use of low$\mathrm{keV}$ virtual monochromatic images may be also limited in patients with large body size [10], the VMI was able to provide virtual high $\mathrm{keV}$ photon images from spectral data as well as low $\mathrm{keV}$ photon images to optimize image quality.

In addition, the lesion conspicuity of hypo- or hyperattenuating FLLs in large phantoms were improved in VMI compared with polychromatic images, especially in low-contrast FLLs. Our study results were also well matched with the results of recent studies which demonstrated improved visualization of hypoattenuating liver lesions [35, 43, 44] or hyperattenuating liver lesions [36] using advanced image-based virtual monochromatic images with a recent dual source DECT system compared with SECT scan. In addition, the lesion conspicuity of low-contrast FLLs was significantly improved in VMI compared to polychromatic images (DRI 16, $P=0.01$; DRI 19, $P=0.02$; DRI 22, $P=0.05$ ), although there was no significant difference among high-contrast FLL images. The improvement of lesion conspicuity in low-contrast FLLs was attributed to effective noise reduction with the use of VMI, suggesting VMI may be an option to differentiate less visible FLLs.

Our study has several limitations we should acknowledge. First, there is a difference between a study phantom and a real human. However, before the clinical application of SDCT, we tried to find out the value of SDCT in obese simulate phantom. Therefore, further studies in real human is strongly warranted. Secondly, we only evaluated the performance of SDCT regarding CNR, image noise, subjective image quality, and FLLs conspicuity. We did not compare 
data with other DECT vendors. Therefore, the comparison with other vendors should be explored in a future study. Third, we used single parameter set in CT acquisition to focus on comparison of conventional and VMI. Thus, further study regarding the influence of CT parameters are strongly warranted.

\section{Conclusion}

VMI of dual-layer spectral detector CT with noise reduction algorithm provides improved CNR, noise reduction, and better subjective image quality in imaging of obese simulated liver phantom compared with polychromatic images. This may hold promise for improving detection of liver lesions and improved imaging of obese patients.

\section{Abbreviations \\ CNR: Contrast to noise ratio; CT: Computed tomography; CTDlvol: Computed tomography dose index volume; DECT: Dual energy computed tomography; DLP: Dose length product; ED: Effective dose; FBP: Filtered back projection; FLL: Focal liver lesion; FOV: Field of view; IR: Iterative reconstruction; keV: Kiloelectronvolt; kVp: Kilovoltage peak; mAs: Milliampere-second; SDCT: Dual-layer spectral detector computed tomography; VMI: Virtual monochromatic spectral image}

\section{Acknowledgements}

We thank to Sung-Ju Lee for his technical assistant.

\section{Author's contributions}

All authors (HJK, JML, SML, HKY, RHK, JGN, AK and JKH) have made substantial contributions to acquisition of data, or analysis and interpretation of data. They have been involved in drafting the manuscript or revising it critically for important intellectual content. The authors have given final approval of the version to be published and agreed to be accountable for all aspects of the work in ensuring that questions related to the accuracy or integrity of any part of the work are appropriately investigated and resolved. Each author - namely HJK, JML, SML, HKY, RHK, JGN, AK and JKH - has participated sufficiently in the work to take public responsibility for appropriate portions of the content.

\section{Funding}

This research did not receive any specific grant from funding agencies in the public, commercial, or not-for-profit sectors.

\section{Availability of data and materials}

The datasets analysed during the current study are available from the corresponding author on reasonable request.

\section{Ethics approval and consent to participate}

Not applicable

\section{Consent for publication}

Not applicable

\section{Competing interests}

The authors declare that they have no competing interests.

\section{Author details}

'Department of Radiology, Seoul National University Hospital, Seoul 03080, South Korea. ${ }^{2}$ Department of Radiology, Seoul National University College of Medicine, 101 Daehangno, Jongno-gu, Seoul 03080, South Korea. Institute of Radiation Medicine, Seoul National University College Medical Research Center, Seoul 03080, South Korea. ${ }^{4}$ Department of Radiology, Hallym University Sacred Heart Hospital, Anyang 14068, South Korea. ${ }^{5}$ Department of Medical imaging, Toronto General hospital, Toronto, Canada. ${ }^{6}$ Department of Radiology, Nizam's Institute of Medical Sciences, Hyderabad 500082, India.
Received: 10 January 2019 Accepted: 5 August 2019

Published online: 28 August 2019

\section{References}

1. Marin D, Boll DT, Mileto A, Nelson RC. State of the art: dual-energy CT of the abdomen. Radiology. 2014;271(2):327-42.

2. Darras KE, McLaughlin PD, Kang H, Black B, Walshe T, Chang SD, Harris AC, Nicolaou S. Virtual monoenergetic reconstruction of contrastenhanced dual energy $\mathrm{CT}$ at $70 \mathrm{keV}$ maximizes mural enhancement in acute small bowel obstruction. Eur J Radiol. 2016;85(5):950-6.

3. Matsumoto K, Jinzaki M, Tanami Y, Ueno A, Yamada M, Kuribayashi S. Virtual monochromatic spectral imaging with fast kilovoltage switching: improved image quality as compared with that obtained with conventional $120-\mathrm{kVp}$ CT. Radiology. 2011;259(1):257-62.

4. Mileto A, Nelson RC, Samei E, Choudhury KR, Jaffe TA, Wilson JM, Marin D. Dual-energy MDCT in hypervascular liver tumors: effect of body size on selection of the optimal monochromatic energy level. Am J Roentgenol. 2014;203(6):1257-64.

5. Agrawal MD, Pinho DF, Kulkarni NM, Hahn PF, Guimaraes AR, Sahani DV. Oncologic applications of dual-energy $C T$ in the abdomen. Radiographics. 2014;34(3):589-612

6. Goo HW, Goo JM. Dual-energy CT: new horizon in medical imaging. Korean J Radiol. 2017;18(4):555-69.

7. Morgan DE. Dual-energy CT of the abdomen. Abdom Imaging. 2014;39(1): $108-34$

8. Heye T, Nelson RC, Ho LM, Marin D, Boll DT. Dual-energy CT applications in the abdomen. AJR Am J Roentgenol. 2012;199(5 Suppl):S64-70,

9. Yeh BM, Shepherd JA, Wang ZJ, Teh HS, Hartman RP, Prevrhal S. Dualenergy and low-kVp CT in the abdomen. AJR Am J Roentgenol. 2009; 193(1):47-54.

10. Yu L, Leng S, McCollough CH. Dual-energy CT-based monochromatic imaging. Am J Roentgenol. 2012;199(5_supplement):S9-S15.

11. Albrecht MH, Scholtz JE, Husers $K$, Beeres M, Bucher AM, Kaup M, Martin SS, Fischer S, Bodelle B, Bauer RW, et al. Advanced image-based virtual monoenergetic dual-energy $C T$ angiography of the abdomen: optimization of kiloelectron volt settings to improve image contrast. Eur Radiol. 2016:26(6):1863-70.

12. Maass C, Baer M, Kachelriess M. Image-based dual energy CT using optimized precorrection functions: a practical new approach of material decomposition in image domain. Med Phys. 2009;36(8):3818-29.

13. Lv P, Lin XZ, Chen K, Gao J. Spectral CT in patients with small HCC: investigation of image quality and diagnostic accuracy. Eur Radiol. 2012; 22(10):2117-24.

14. Kim KS, Lee JM, Kim SH, Kim KW, Kim SJ, Cho SH, Han JK, Choi BI. Image fusion in dual energy computed tomography for detection of hypervascular liver hepatocellular carcinoma: phantom and preliminary studies. Investig Radiol. 2010;45(3):149-57.

15. Grant KL, Flohr TG, Krauss B, Sedlmair M, Thomas C, Schmidt B. Assessment of an advanced image-based technique to calculate virtual monoenergetic computed tomographic images from a dualenergy examination to improve contrast-to-noise ratio in examinations using iodinated contrast media. Investig Radiol. 2014; 49(9):586-92

16. Sun H, Qiu S, Lou S, Liu J, Li C, Jiang G. A correction method for nonlinear artifacts in CT imaging. In: Engineering in Medicine and Biology Society, 2004 IEMBS'04 26th Annual International Conference of the IEEE: 2004: IEEE; 2004. p. 1290-3.

17. Brooks RA, Di Chiro G. Beam hardening in x-ray reconstructive tomography. Phys Med Biol. 1976;21(3):390.

18. Menvielle N, Goussard Y, Orban D, Soulez G. Reduction of beamhardening artifacts in X-ray CT. In: 2005 IEEE Engineering in Medicine and Biology 27th Annual Conference: 2005: IEEE; 2005. p. 1865-8.

19. Jacobsen M, Wood C, Cody D. WE-FG-207B-08: dual-energy CT iodine accuracy across vendors and platforms. Med Phys. 2016;43(6):3835-6.

20. Romman Z, Uman I, Yagil Y, Finizi D, Wainer N, Milstein D: Detector technology in simultaneous spectral imaging. Philips White Paper, available online 2015, 29

21. Ozguner O, Dhanantwari A, Halliburton S, Wen G, Utrup S, Jordan D. SU-G-leP2-09: iodine imaging at spectral CT with a dual-layer detector. Med Phys. 2016;43(6):3657. 
22. Duan X, Arbique G, Guild J, Yagil Y, Anderson J. WE-FG-207B-12: quantitative evaluation of a spectral CT scanner in a phantom study: results of spectral reconstructions. Med Phys. 2016;43(6):3836-7.

23. Jacobsen M, Wood C, Cody D. WE-FG-207B-10: dual-energy CT monochromatic image consistency across vendors and platforms. Med Phys. 2016:43(6):3836.

24. Sellerer T, Ehn S, Hammel J, Noel PB, Fingerle AA, Patino M, Parakh A, Sahani DV, Zeiter S, Holz JA. Dual-energy CT. A phantom comparison of different platforms for abdominal imaging. Eur Radiol. 2018;28(7):2745-55.

25. Ehn S, Sellerer T, Muenzel D, Fingerle A, Kopp F, Duda M, Mei K, Renger B, Herzen J, Dangelmaier J. Assessment of quantification accuracy and image quality of a full-body dual-layer spectral CT system. J Appl Clin Med Phys. 2018;19(1):204-17.

26. Yoon JH, Lee JM, Yu MH, Baek JH, Jeon JH, Hur BY, Dhanantwari A, Chung SY, Han JK, Choi BI. Comparison of iterative model-based reconstruction versus conventional filtered Back projection and hybrid iterative reconstruction techniques: lesion conspicuity and influence of body size in anthropomorphic liver phantoms. J Comput Assist Tomogr 2014;38(6):859-68

27. Ananthakrishnan L, Rajiah P, Ahn R, Rassouli N, Xi Y, Soesbe TC, Lewis MA, Lenkinski RE, Leyendecker JR, Abbara S. Spectral detector CT-derived virtual non-contrast images: comparison of attenuation values with unenhanced CT. Abdom Radiol. 2017;42(3):702.

28. Park HJ, Lee JM, Park SB, Lee JB, Jeong YK, Yoon JH. Comparison of knowledge-based iterative model reconstruction and hybrid reconstruction techniques for liver CT evaluation of Hypervascular hepatocellular carcinoma. J Comput Assist Tomogr. 2016;40(6):863-71.

29. Bongartz G, Golding S, Jurik A, Leonardi M, Van Persijn Van Meerten E, Rodríguez R, Schneider K, Calzado A, Geleijns J, Jessen K. European guidelines for multislice computed tomography. Brussels: European Commission; 2004. http://biophysicssite.com/html/msct_quality_criteria_2 004.html.

30. Pickhardt PJ, Lubner MG, Kim DH, Tang J, Ruma JA, del Rio AM, Chen G-H. Abdominal CT with model-based iterative reconstruction (MBIR): initial results of a prospective trial comparing ultralow-dose with standard-dose imaging. AJR Am J Roentgenol. 2012;199(6):1266.

31. Yu MH, Lee JM, Yoon J-H, Baek JH, Han JK, Choi B-I, Flohr TG. Low tube voltage intermediate tube current liver MDCT: sinogram-affirmed iterative reconstruction algorithm for detection of hypervascular hepatocellular carcinoma. Am J Roentgenol. 2013;201(1):23-32.

32. Yamada $Y$, Jinzaki M, Hosokawa T, Tanami $Y$, Abe T, Kuribayashi S. Abdominal CT: an intra-individual comparison between virtual monochromatic spectral and polychromatic 120-kVp images obtained during the same examination. Eur J Radiol. 2014;83(10):1715-22.

33. Yu L, Christner JA, Leng S, Wang J, Fletcher JG, McCollough CH. Virtual monochromatic imaging in dual-source dual-energy $C T$ : radiation dose and image quality. Med Phys. 2011;38(12):6371-9.

34. Leng S, Yu L, Fletcher JG, McCollough CH. Maximizing iodine contrast-tonoise ratios in abdominal $\mathrm{CT}$ imaging through use of energy domain noise reduction and virtual Monoenergetic dual-energy CT. Radiology. 2015: 276(2):562-70

35. Caruso D, De Cecco CN, Schoepf UJ, Schaefer AR, Leland PW, Johnson D, Laghi A, Hardie AD. Can dual-energy computed tomography improve visualization of hypoenhancing liver lesions in portal venous phase? Assessment of advanced image-based virtual monoenergetic images. Clin Imaging. 2017;41:118-24.

36. Husarik DB, Gordic S, Desbiolles L, Krauss B, Leschka S, Wildermuth S, Alkadhi $\mathrm{H}$. Advanced virtual monoenergetic computed tomography of hyperattenuating and hypoattenuating liver lesions: ex-vivo and patient experience in various body sizes. Investig Radiol. 2015;50(10):695-702.

37. Zhang D, Li X, Liu B. Objective characterization of GE discovery CT750 HD scanner: gemstone spectral imaging mode. Med Phys. 2011;38(3):1178-88.

38. Cui Y, Gao SY, Wang ZL, Li XT, Sun YS, Tang L, Zhang XP. Which should be the routine cross-sectional reconstruction mode in spectral $C T$ imaging: monochromatic or polychromatic? Br J Radiol. 2012;85(1018):e887-90.

39. Chen Y, Liu J, Hu Y, Yang J, Shi L, Shu H, Gui Z, Coatrieux G, Luo L. Discriminative feature representation: an effective postprocessing solution to low dose CT imaging. Phys Med Biol. 2017;62(6):2103.

40. Liu J, Hu Y, Yang J, Chen Y, Shu H, Luo L, Feng Q, Gui Z, Coatrieux G. 3D feature constrained reconstruction for low-dose CT imaging. IEEE Transactions on Circuits and Systems for Video Technology. 2018;28(5):1232-47.
41. Chen Y, Shi L, Feng Q, Yang J, Shu H, Luo L, Coatrieux J-L, Chen W. Artifact suppressed dictionary learning for low-dose $\mathrm{CT}$ image processing. IEEE Trans Med Imaging. 2014;33(12):2271-92.

42. Mileto A, Barina A, Marin D, Stinnett SS, Roy Choudhury K, Wilson JM, Nelson RC. Virtual monochromatic images from dual-energy multidetector $C T$ : variance in $C T$ numbers from the same lesion between single-source projection-based and dual-source image-based implementations. Radiology. 2016;279(1):269-77.

43. Robinson E, Babb J, Chandarana H, Macari M. Dual source dual energy MDCT: comparison of $80 \mathrm{kVp}$ and weighted average $120 \mathrm{kVp}$ data for conspicuity of hypo-vascular liver metastases. Investig Radiol. 2010;45(7):413-8.

44. Hokamp NG, Obmann V, Kessner R, Laukamp K, Persigehl T, Haneder S, Ramaiya N. Improved visualization of hypodense liver lesions in virtual monoenergetic images from spectral detector CT: proof of concept in a 3Dprinted phantom and evaluation in 74 patients. Eur J Radiol. 2018;109:114-23.

\section{Publisher's Note}

Springer Nature remains neutral with regard to jurisdictional claims in published maps and institutional affiliations.

Ready to submit your research? Choose BMC and benefit from:

- fast, convenient online submission

- thorough peer review by experienced researchers in your field

- rapid publication on acceptance

- support for research data, including large and complex data types

- gold Open Access which fosters wider collaboration and increased citations

- maximum visibility for your research: over $100 \mathrm{M}$ website views per year

At BMC, research is always in progress.

Learn more biomedcentral.com/submissions 